\title{
Caracterização de Diferentes Amostras de Mandioca por Espectroscopia de Ressonância Magnética Nuclear
}

\author{
Gisele C. V. Iulianelli, Maria I. B. Tavares \\ Instituto de Macromoléculas Professora Eloísa Mano, UFRJ
}

\begin{abstract}
Resumo: A raiz de mandioca (Manihot esculenta crantz) é cultivada nas mais diversas regiões do Brasil e desempenha importante papel na dieta alimentar dos brasileiros, representando para muitas famílias do Norte e Nordeste a principal fonte energética. A farinha de mandioca constitui-se num alimento com alto teor de amido, além de conter fibras, lipídeos e alguns minerais, entretanto apresenta grande variabilidade genética, o que resulta em diferenciação na sua constituição química e estrutural. Frente à importância econômica, nutricional e farmacológica deste produto, o presente trabalho objetivou caracterizar seis diferentes amostras de farinha de mandioca por meio da espectroscopia de RMN. Além de estabelecer o assinalamento dos principais grupamentos químicos, os resultados confirmaram diferenças no aspecto químico e estrutural das amostras de mandioca. Foi visto que a amostra F1 é mais rica em carboidratos, a amostra F4 é mais rica em glicolipídeos, a amostra F2 apresenta alto teor de amilose, e a amostra F6 apresenta maior diversidade de glicolipídeos. Em relação à estrutura molecular, os espectros de RMN indicaram que a amostra F1 apresenta maior organização molecular e que as amostras F3 e F5 apresentam similaridade na amorficidade e também no arranjo e empacotamento molecular.
\end{abstract}

Palavras-chave: RMN, mandioca, caracterização química e estrutural.

\section{Characterization of Different Cassava Samples by Nuclear Magnetic Resonance Spectroscopy}

\begin{abstract}
Cassava root (Manihot esculenta crantz) is grown in all Brazilian states, being an important product in the diet of Brazilians. For many families of the North and Northeast states, it may represent the main energy source. The cassava root flour has high levels of starch, in addition to containing fiber, lipids and some minerals. There is, however, great genetic variability, which results in differentiation in its chemical composition and structural aspect. Motivated by the economic, nutritional and pharmacological importance of this product, this work is aimed at characterizing six cassava flour samples by NMR spectroscopy. The spectra revealed the main chemical groups. Furthermore, the results confirmed differences on chemical and structural aspect of the samples. For instance, the F1 sample is richer in carbohydrates, while the F4 sample has higher proportion of glycolipids, the F2 sample has higher amylose content and the F6 sample exhibits a greater diversity of glycolipid types. Regarding the molecular structure, the NMR spectra indicated that the F1 sample is more organized at the molecular level, while the F3 and F5 samples are similar in amorphicity and in the molecular packing.
\end{abstract}

Keywords: NMR, cassava, chemical and structural characterization.

\section{Introdução}

O cultivo da mandioca é de grande relevância econômica por representar a principal fonte de carboidratos para milhões de pessoas, essencialmente nos países em desenvolvimento como no Brasil, que é o segundo maior produtor mundial de mandioca e atribui a este produto uma grande importância na alimentação humana e animal, como matéria prima para inúmeros produtos industriais e na geração de emprego e renda ${ }^{[1-3]}$.

No entanto, a cultura da mandioca apresenta uma grande variabilidade, a raiz é constituída essencialmente por amido, umidade, fibra alimentar, proteínas, lipídios e minerais como potássio, cálcio, fósforo, sódio e ferro ${ }^{[4-6]}$. Enquanto o amido é a fonte mais importante de carboidratos na alimentação humana, representando 80-90\% de todos os polissacarídeos da dieta com grande valor nutricional ${ }^{[7]}$, a fibra desempenha um importante papel fisiológico na regulação do funcionamento do trato gastrointestinal, assim como no controle e/ou prevenção de doenças crônicas e degenerativas ${ }^{[8]}$. Adicionalmente, existem fortes indícios de que essas fibras podem desempenhar um efeito protetor contra o câncer de cólon e doenças cardíacas ${ }^{[9]}$. Contudo, é sabido que o percentual dos elementos que compõe a raiz de mandioca pode diferir substancialmente de uma espécie para outra, resultando em diferenças singulares relacionadas tanto às características próprias como gosto, tempo de cozimento, modo de uso e processo de estocagem, como também no aspecto nutricional e farmacológico em função da composição química apresentada por cada variedade.

No Brasil, apesar da mandioca representar a base alimentar de grande parte da sua população $0^{[10]}$, poucos estudos têm sido conduzidos com o objetivo de comparar as diferentes variedades de mandioca quanto ao aspecto químico e estrutural, informações estas, valiosíssimas no que tange a sua qualidade, valor nutricional e aplicabilidade.

A espectroscopia de Ressonância Magnética Nuclear é conhecida como uma importante ferramenta analítica na caracterização de materiais heterogêneos de fontes naturais, como é o caso de alimentos como a mandioca. A RMN apresenta disponibilidade de técnicas diversas para caracterizar esses materiais e pode ser dividida em espectroscopia de alta resolução (alto campo magnético) e baixa resolução (baixo campo magnético). Dentre os principais elementos que compõem os alimentos, os isótopos de ${ }^{1} \mathrm{H},{ }^{13} \mathrm{C}$ e ${ }^{31} \mathrm{P}$ são os mais usados nesses estudos devido à abundância natural e sensibilidade destes núcleos. Técnicas de RMN de alta resolução como ${ }^{13} \mathrm{C}$, APT e ${ }^{1} \mathrm{H}$ representam uma excelente alternativa para o estudo de alimentos devido à simplicidade de execução e à importância das informações geradas. As técnicas de ${ }^{13} \mathrm{C}$ e ${ }^{1} \mathrm{H}$ são técnicas diretas de observação do sinal destes núcleos, chamadas técnicas de pulso simples, onde aplica-se um pulso de $90^{\circ}$ e adquire-se o sinal. APT (Attached

Autor para correspondência: Gisele C. V. Iulianelli, Instituto de Macromoléculas Professora Eloísa Mano, Universidade Federal do Rio de Janeiro,

Cidade Universitária, Bloco J, Ilha do Fundão, Caixa Postal 68525, CEP: 21945-970, Rio de Janeiro, RJ, Brasil. E-mail: gisele@ima.ufrj.br 
Proton Test) é uma técnica de simplificação espectral. A sequência de pulsos empregada na execução da técnica APT tem como base a modulação por eco de spin da constante de acoplamento C-H e envolve as etapas de preparação, evolução e detecção. Esta sequência de pulsos pode ser resumida da seguinte maneira: aplica-se um pulso de $90^{\circ}$, desliga-se o desacoplador, aplica-se um pulso de $180^{\circ}$, e por fim religa-se o desacoplador. Por meio desta sequência de pulso é possível separar os sinais dos carbonos de acordo com o número de hidrogênios ligados a ele, de modo que os carbonos que possuem números ímpares de hidrogênio $\left(\mathrm{CH}_{3} \mathrm{e} \mathrm{CH}\right)$ são detectados em antifase (invertidos no espectro) e os que possuem números pares de hidrogênio ou os carbonos não hidrogenados (como por exemplo $\mathrm{CH}_{2}$ e carbonilas) são detectados em fase, ou seja, mostram sinais de ressonância não invertidos no espectro ${ }^{[11,12]}$.

A proposta deste trabalho é caracterizar seis diferentes variedades de farinha de mandioca (Manihot esculenta), comparálas e diferenciá-las quanto ao aspecto químico e ordenação molecular, empregando técnicas de Ressonância Magnética Nuclear de alto campo magnético pelo estudo dos núcleos de ${ }^{13} \mathrm{C}$ e ${ }^{1} \mathrm{H}$.

\section{Experimental}

As variedades de mandioca escolhidas para o desenvolvimento deste estudo foram cedidas pela EMBRAPA e são provenientes da região amazônica, mais especificamente do Estado do Pará. Esta escolha advém do fato desta localidade ser rica em uma ampla variedade de espécies dessa raiz e se destacar como principal produtor nacional de mandioca. As variedades de farinha de mandioca submetidas à caracterização estão representadas pelas siglas: F1, F2, F3, F4, F5 e F6.

Para alcançar a proposta deste trabalho foram realizadas análises de Ressonância Magnética Nuclear (RMN) em solução utilizando as técnicas de ${ }^{13} \mathrm{C},{ }^{1} \mathrm{H}$ e APT. O equipamento utilizado foi um espectrômetro de $300 \mathrm{MHz}$ (Varian Mercury 300). O procedimento empregado para a obtenção dos espectros e os parâmetros necessários para cada técnica de análise estão descritos a seguir:

Para análise do núcleo de ${ }^{13} \mathrm{C}$ foram preparadas soluções de farinha de mandioca utilizando aproximadamente $100 \mathrm{mg}$ de amostra e $2 \mathrm{~mL}$ de água deuterada $\left(\mathrm{D}_{2} \mathrm{O}\right)$. As amostras foram colocadas em tubos de RMN de $10 \mathrm{~mm}$ de diâmetro e posteriormente na sonda de $10 \mathrm{~mm}$. Como marcador empregou-se o TSP (Trimethyl Silyl Propionate).

Para análise do núcleo de ${ }^{1} \mathrm{H}$ foram preparadas soluções de farinha de mandioca utilizando aproximadamente $20 \mathrm{mg}$ de amostra e 0,6 mL de água deuterada $\left(\mathrm{D}_{2} \mathrm{O}\right)$. As amostras foram colocadas em tubos de RMN de $5 \mathrm{~mm}$ de diâmetro e posteriormente na sonda de $5 \mathrm{~mm}$.

Os parâmetros empregados para análise dos núcleos em observação estão relacionados na Tabela 1 .

Tabela 1. Parâmetros utilizados para obtenção dos espectros de RMN.

\begin{tabular}{ccc}
\hline Parâmetros & Valores $\left({ }^{13} \mathbf{C}\right.$ e APT) & Valores $\left({ }^{1} \mathbf{H}\right)$ \\
\hline $\begin{array}{c}\text { Frequência de observação } \\
(\mathrm{MHz})\end{array}$ & 75 & 300 \\
$\begin{array}{c}\text { Tempo de aquisição } \\
\quad \text { (segundos) } \\
\text { Janela espectral } \\
(\mathrm{Hz})\end{array}$ & 1,8 & 3,3 \\
$\begin{array}{c}\text { Largura do pulso }(\mu \mathrm{s}) \\
\left(90^{\circ}\right)\end{array}$ & 18000 & 4800 \\
$\begin{array}{c}\text { Intervalo entre os pulsos } \\
(\text { segundos })\end{array}$ & 1 & 14,7 \\
Número de acúmulos & 16000 & 128 \\
\hline
\end{tabular}

\section{Resultados e Discussão}

As técnicas de ${ }^{13} \mathrm{C}$ e APT são complementares, visto que o APT é uma técnica de simplificação espectral utilizado estritamente para a diferenciação dos sinais de $\mathrm{CH}$ e $\mathrm{CH}_{2}$, que muitas vezes podem estar sobrepostos nos espectros de ${ }^{13} \mathrm{C}$, sendo empregada deste modo para auxiliar na determinação dos deslocamentos químicos.

Os espectros de RMN de ${ }^{13} \mathrm{C}$ e APT obtidos para as seis variedades de mandiocas estudadas estão mostrados na Figura 1 e Figura 2, respectivamente. A Tabela 2 mostra os deslocamentos químicos observados nos espectros para as diferentes amostras de mandioca e a Figura 3 ilustra os carbonos referentes à estrutura do amido presente na mandioca (C-1, C-2, C-3, C-4, C-5 e C-6).

Os espectros de ${ }^{13} \mathrm{C}$ das amostras F1, F2, F3, F5 e F6 (Figura 1) mostram três faixas definidas de sinais. A primeira localizada aproximadamente entre 63 e 67 ppm está relacionada aos grupamentos $\mathrm{CH}_{2}-\mathrm{OH}$ (C-6); uma outra faixa entre 70 e 84 ppm aproximadamente representa os grupos $\mathrm{CH}-\mathrm{O}(\mathrm{C}-2, \mathrm{C}-3, \mathrm{C}-4$ e C-5), e por fim a faixa localizada aproximadamente entre 94 e 107 ppm é referente ao grupamento $\mathrm{C}-\mathrm{O}-\mathrm{C}$ dos carbonos anoméricos $(\mathrm{C}-1)^{[9,14-16]}$. O espectro de ${ }^{13} \mathrm{C}$ da amostra $\mathrm{F} 4$ apresentou, além das três faixas de sinais mencionadas para as demais amostras, uma outra faixa de sinais entre 19,4 e 25,3 ppm. Esses sinais são referentes aos carbonos oriundos de glicolipídeos e sinalizam que esta amostra apresenta uma riqueza maior deste componente.

As amostras F2 a F6 apresentaram perfis similares conforme apresentado pelos espectros de ${ }^{13} \mathrm{C}$ (Figura 1) e corroborado pelos espectros de APT (Figura 2). Estas amostras são compostas majoritariamente pelos polissacarídeos amilose e amilopectina como indicado pela detecção de apenas um sinal localizado em 102 ppm referente ao $\mathrm{C}$ anomérico destes polissacarídeos. Por outro lado os espectros de ${ }^{13} \mathrm{C}$ e APT obtidos para a amostra F1 mostram uma expressiva diferenciação nesta região. A detecção de seis sinais contidos na faixa do carbono anomérico deve-se a ligações de diferentes sequenciamentos dos polissacarídeos. O número mais expressivo de sinais contidos nesta região sinaliza que a amostra F1 apresenta uma maior diversidade de carboidratos. $\mathrm{O}$ espectro de APT corrobora esta constatação por meio da detecção de 3 sinais invertidos nesta mesma região, indicando a presença de pelo menos dois polissacarídeos diferentes da amilose e da amilopectina. É sabido que devido à semelhança estrutural entre esses polissacarídeos não é possível distingui-los por RMN em solução, entretanto foi detectada a presença de polissacarídeos de cadeias menores em 98 e $94 \mathrm{ppm}$. Adicionalmente, o espectro de ${ }^{13} \mathrm{C}$ da amostra F1 mostra sinais localizados em 83,4 e 84 ppm referentes ao C-4 da região cristalina, indicando que esta amostra apresenta regiões expressivas de ordenação molecular propiciada pela cristalinidade, o que provavelmente está relacionado à amilopectina, já que esta estrutura é responsável pela cristalinidade nos grânulos de amido ${ }^{[16]}$.

Os deslocamentos químicos localizados em aproximadamente $0,17,21$ e 57 ppm presentes em todas as amostras são concernentes ao marcador utilizado (TSP).

Os espectros de RMN de ${ }^{1} \mathrm{H}$ obtidos para as seis variedades de mandiocas estudadas estão mostrados na Figura 4. A Tabela 3 mostra os deslocamentos químicos observados a partir desses espectros. De acordo com os espectros obtidos, as amostras F3 e F4 apresentam sinais em três faixas e as amostras F1, F2, F5 e F6 mostram sinais em quatro faixas específicas como destacado na Tabela 3. Os sinais localizados entre 1,3 e 2,8 ppm são referentes aos ${ }^{1} \mathrm{H}$ de lipídeos presentes na amostra, os sinais de ${ }^{1} \mathrm{H}$ ligados à heteroátomos $(\mathrm{OH})$ encontram-se entre 3,4 e 4,6 ppm, e por último os sinais entre $5 \mathrm{ppm}$ e 5,8 são referentes aos ${ }^{1} \mathrm{H}$ ligados ao carbono anomérico ${ }^{[9,17,18]}$. $\mathrm{O}$ número significativo de sinais referentes aos ${ }^{1} \mathrm{H}$ ligados ao carbono anomérico encontrados para a amostra F1 indica que esta 


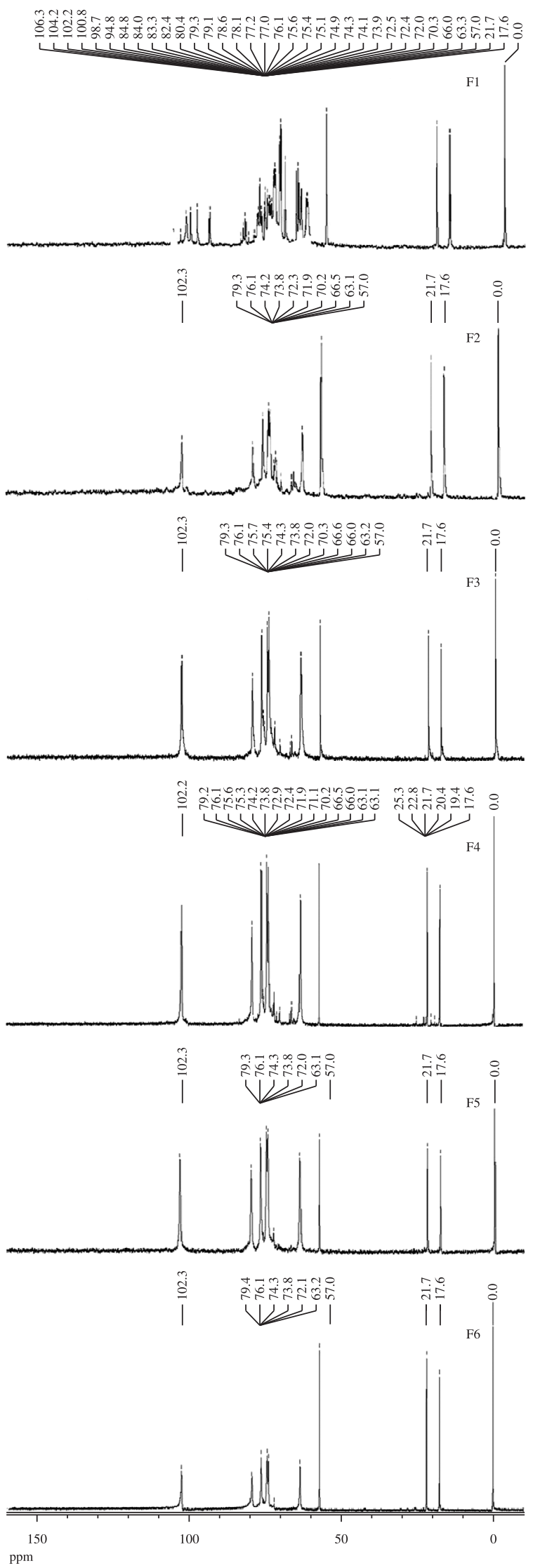

Figura 1. Espectro de $\mathrm{RMN}$ de ${ }^{13} \mathrm{C}$ das diferentes variedades de mandioca.

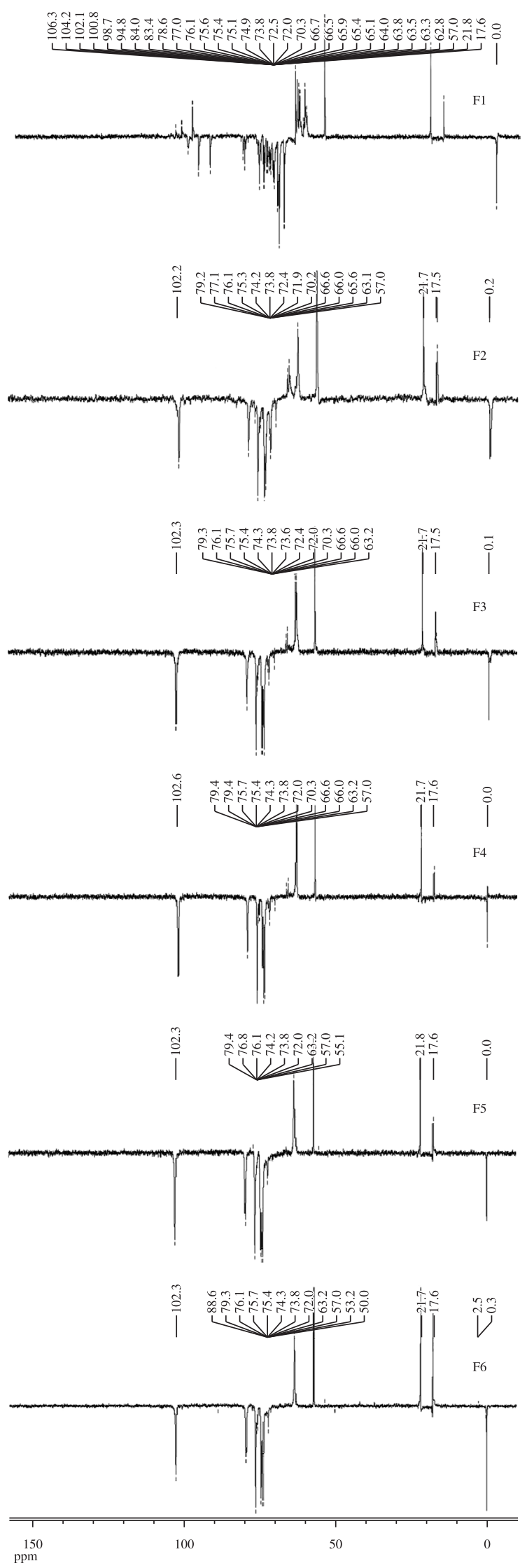

Figura 2. Espectro de RMN APT das diferentes variedades de mandioca. 
Tabela 2. Deslocamentos químicos observados no espectro de RMN de ${ }^{13} \mathrm{C}$ em solução para as seis variedades de mandiocas.

\begin{tabular}{|c|c|c|c|c|c|c|}
\hline \multirow[t]{2}{*}{ Carbonos } & \multicolumn{6}{|c|}{$\begin{array}{c}\text { Deslocamentos químicos (ppm) das } \\
\text { amostras de mandioca }\end{array}$} \\
\hline & F1 & F2 & F3 & F4 & F5 & F6 \\
\hline \multirow[t]{6}{*}{$\mathrm{C}-1$} & 106,3 & 102,3 & 102,3 & 102,2 & 102,3 & 102,3 \\
\hline & 104,2 & & & & & \\
\hline & 102,2 & & & & & \\
\hline & 100,8 & & & & & \\
\hline & 98,7 & & & & & \\
\hline & 94,8 & & & & & \\
\hline \multirow[t]{15}{*}{ C-4 } & 84,8 & 79,3 & 79,3 & 79,2 & 79,3 & 79,4 \\
\hline & 84,0 & 76,1 & 76,1 & 76,1 & 76,1 & 76,1 \\
\hline & 83,3 & & 75,7 & 75,6 & & \\
\hline & 82,4 & & 75,4 & 75,3 & & \\
\hline & 80,0 & & & & & \\
\hline & 79,3 & & & & & \\
\hline & 79,1 & & & & & \\
\hline & 78,6 & & & & & \\
\hline & 78,1 & & & & & \\
\hline & 77,2 & & & & & \\
\hline & 77,0 & & & & & \\
\hline & 76,1 & & & & & \\
\hline & 75,6 & & & & & \\
\hline & 75,4 & & & & & \\
\hline & 75,1 & & & & & \\
\hline \multirow[t]{8}{*}{$\mathrm{C}-5, \mathrm{C}-3$ e C-2 } & 74,9 & 74,2 & 74,3 & 74,2 & 74,3 & 74,3 \\
\hline & 74,1 & 73,8 & 73,8 & 73,8 & 73,8 & 73,8 \\
\hline & 73,9 & 72,3 & 72,0 & 72,4 & 72,0 & 72,1 \\
\hline & 73,6 & 71,9 & 70,3 & 71,9 & & \\
\hline & 72,5 & 70,2 & & 71,1 & & \\
\hline & 72,4 & & & 70,2 & & \\
\hline & 72,0 & & & & & \\
\hline & 70,3 & & & & & \\
\hline \multirow[t]{3}{*}{ C-6 } & 66,0 & 66,5 & 66,6 & 66,5 & 63,1 & 63,2 \\
\hline & 63,3 & 63,1 & 66,0 & 66,0 & & \\
\hline & & & 63,2 & 63,1 & & \\
\hline \multirow[t]{4}{*}{ Marcador (TSP) } & 57,0 & 57,0 & 57,0 & 57,0 & 57,0 & 57,0 \\
\hline & 21,7 & 21,7 & 21,7 & 21,7 & 21,7 & 21,7 \\
\hline & 17,6 & 17,6 & 17,6 & 17,6 & 17,6 & 17,6 \\
\hline & 0 & 0 & 0 & 0 & 0 & 0 \\
\hline \multirow{4}{*}{$\begin{array}{l}\text { Carbonos de } \\
\text { glicolipídeos }\end{array}$} & & & & 25,3 & & \\
\hline & & & & 22,8 & & \\
\hline & & & & 20,4 & & \\
\hline & & & & 19,4 & & \\
\hline
\end{tabular}

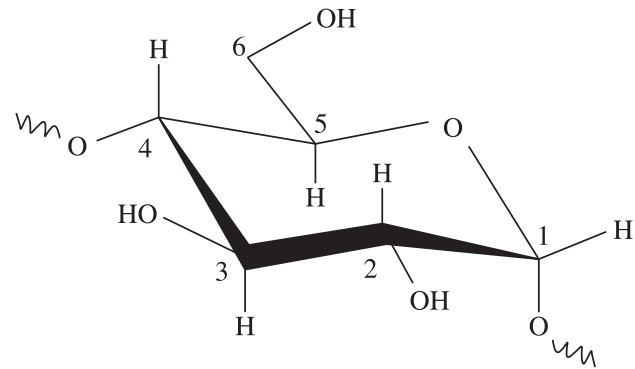

Figura 3. Estrutura básica do amido, mostrando os carbonos correspondentes ${ }^{[13]}$.

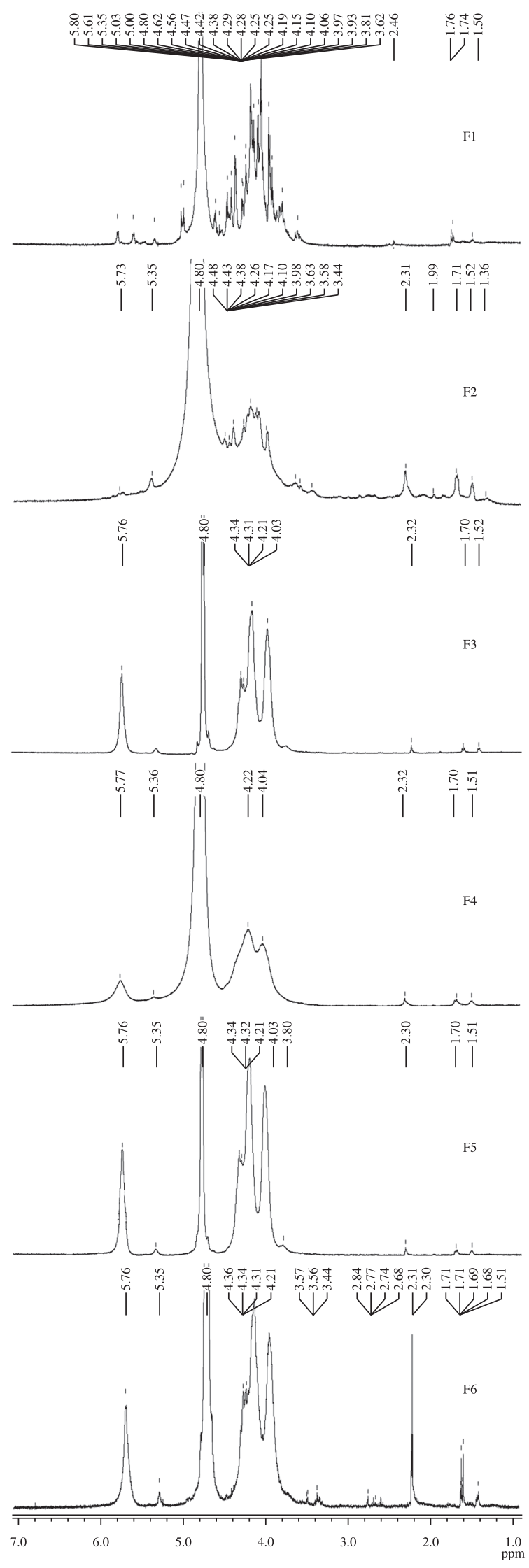

Figura 4. Espectro de $\mathrm{RMN}$ de ${ }^{1} \mathrm{H}$ das diferentes variedades de mandioca. 
Tabela 3. Deslocamentos químicos observados no espectro de $\mathrm{RMN}$ de ${ }^{1} \mathrm{H}$ para as seis variedades de mandiocas.

\begin{tabular}{|c|c|c|c|c|c|c|}
\hline \multirow[t]{2}{*}{ Hidrogênios } & \multicolumn{6}{|c|}{$\begin{array}{c}\text { Deslocamentos químicos (ppm) das amostras } \\
\text { de mandioca }\end{array}$} \\
\hline & F1 & F2 & F3 & F4 & F5 & F6 \\
\hline \multirow{5}{*}{$\begin{array}{l}{ }^{1} \mathrm{H} \text { ligados } \\
\text { ao carbono } \\
\text { anomérico }\end{array}$} & 5,8 & 5,7 & 5,7 & 5,7 & 5,7 & 5,7 \\
\hline & 5,6 & 5,3 & & 5,3 & 5,3 & 5,3 \\
\hline & 5,3 & & & & & \\
\hline & 5,0 & & & & & \\
\hline & 5,0 & & & & & \\
\hline Marcador (HOD) & 4,8 & 4,8 & 4,8 & 4,8 & 4,8 & 4,8 \\
\hline \multirow{16}{*}{$\begin{array}{l}{ }^{1} \mathrm{H} \text { ligados à } \\
\text { heteroátomos }\end{array}$} & 4,6 & 4,4 & 4,3 & 4,2 & 4,3 & 4,3 \\
\hline & 4,5 & 4,4 & 4,3 & 4,0 & 4,3 & 4,3 \\
\hline & 4,4 & 4,3 & 4,2 & & 4,2 & 4,3 \\
\hline & 4,4 & 4,2 & 4,0 & & 4,0 & 4,2 \\
\hline & 4,3 & 4,1 & & & 3,8 & 3,5 \\
\hline & 4,2 & 4,1 & & & & 3,5 \\
\hline & 4,2 & 3,9 & & & & 3,4 \\
\hline & 4,2 & 3,6 & & & & \\
\hline & 4,1 & 3,5 & & & & \\
\hline & 4,1 & 3,4 & & & & \\
\hline & 4,1 & & & & & \\
\hline & 4,0 & & & & & \\
\hline & 3,9 & & & & & \\
\hline & 3,9 & & & & & \\
\hline & 3,8 & & & & & \\
\hline & 3,6 & & & & & \\
\hline \multirow[t]{11}{*}{${ }^{1} \mathrm{H}$ de lipídeos } & 2,4 & 2,3 & 2,3 & 2,3 & 2,3 & 2,8 \\
\hline & 1,7 & 1,9 & 1,7 & 1,7 & 1,7 & 2,7 \\
\hline & 1,7 & 1,7 & 1,5 & 1,5 & 1,5 & 2,7 \\
\hline & 1,5 & 1,5 & & & & 2,6 \\
\hline & & 1,3 & & & & 2,3 \\
\hline & & & & & & 2,3 \\
\hline & & & & & & 1,7 \\
\hline & & & & & & 1,7 \\
\hline & & & & & & 1,6 \\
\hline & & & & & & 1,6 \\
\hline & & & & & & 1,5 \\
\hline
\end{tabular}

amostra apresenta outros tipos de carboidratos além da amilose e amilopectina, corroborando os resultados obtidos pelas técnicas de ${ }^{13} \mathrm{C}$ e APT. O alargamento dos sinais observados no espectro de RMN para a amostra F2 (Figura 4) indica uma amorficidade expressiva, que provavelmente está relacionada ao alto teor de amilose presente na amostra. O número expressivo de sinais localizados na faixa referente aos ${ }^{1} \mathrm{H}$ de lipídeos sinaliza que a amostra F6 apresenta uma maior diversidade no tipo de lipídeos presentes.

Apesar da faixa de detecção/observação do espectro de RMN de ${ }^{1} \mathrm{H}$ ser muito menor do que a faixa do ${ }^{13} \mathrm{C}$, o que prejudica a resolução do espectro em face da distribuição dos sinais dos carboidratos, a observação do perfil dos espectros permitiu importantes constatações. A primeira está relacionada à amostra $\mathrm{F} 1$, que apresentou um espectro com sinais bem mais definidos, indicando que esta amostra apresenta uma maior organização de arranjo molecular comparada às demais amostras de mandioca. A segunda diz respeito às amostras F3 e F5, que além de apresentarem amorficidade significativa, indicado pelo alargamento dos sinais, apresentam similaridade também no que tange ao arranjo e empacotamento molecular, como pode ser comprovado pela semelhança no perfil desses espectros (Figura 4).

\section{Conclusões}

Além de estabelecer o assinalamento dos diferentes e principais grupamentos químicos presentes nas amostras de mandioca, a espectroscopia de Ressonância Magnética Nuclear em solução por meio das técnicas de ${ }^{13} \mathrm{C}$, APT e ${ }^{1} \mathrm{H}$ foi imprescindível para alcançar outras importantes constatações no que diz respeito à estrutura química e molecular das amostras analisadas. Os espectros de ${ }^{13} \mathrm{C}$ e APT mostraram que a variedade de mandioca $F 1$ possui uma maior ordenação molecular que está relacionada principalmente à amilopectina, e também apresenta outros tipos de carboidratos além dos polissacarídeos amilose e amilopectina, sinalizando que a amostra $\mathrm{F} 1$ é mais rica em variedade de carboidratos comparada às demais amostras. Adicionalmente, foi visto que a amostra F4 é mais rica em glicolipídeos. A espectroscopia de $\mathrm{RMN}$ de ${ }^{1} \mathrm{H}$ confirmou a maior diversidade em carboidratos presente na amostra F1; mostrou uma amorficidade expressiva na amostra F2, que está relacionada ao alto teor de amilose presente na amostra; e sinalizou que a amostra F6 apresenta uma maior diversidade no que se refere ao tipo de glicolipídeos presentes. Em relação à estrutura molecular, os espectros de $\mathrm{RMN}$ de ${ }^{1} \mathrm{H}$ indicaram que a amostra $\mathrm{F} 1$ apresenta uma maior organização de arranjo molecular comparada às demais amostras de mandioca, corroborando o resultado encontrado por RMN de ${ }^{13} \mathrm{C}$; e que as amostras F3 e F5 apresentam similaridade na amorficidade e também no arranjo e empacotamento molecular. Quanto ao aspecto nutricional/farmacológico, as amostras F1, F4 e F6 mostraram maior expressividade por apresentarem maior riqueza em lipídeos ou carboidratos.

\section{Agradecimentos}

Os autores agradecem à FAPERJ por financiar este trabalho, à EMBRAPA por ceder as amostras de mandioca para avaliação e ao Laboratório de RMN do IMA/UFRJ.

\section{Referências Bibliográficas}

1. Souza, L. S. \& Fialho, J. F. - "Embrapa Mandioca e Fruticultura. Sistemas de Produção, 8" (2003).

2. Menezes, M. P. C.; Ribeiro, M. N.; Costa, R. G. \& Medeiros, A. N. Rev. Bras. Zoot., 33, p.729 (2004).

3. Diniz, M. S.; Oliveira, A. M. G.; Coutinho, S. C. \& Santana, M. A. "Embrapa Mandioca e Fruticultura, Boletim de Pesquisa e Desenvolvimento, 35" (2004).

4. Dias, L. T. \& Leonel, M. - Ciênc. Agrotec., 30, p.692 (2006).

5. Chijindu, E. N. \& Boateng, B. A. World J. Agric. Sci., 4, p.404 (2008).

6. Souza, J. M. L.; Álvares, V. S.; Leite, F. M. N.; Reis, F. S. \& Felisberto, F. Á. V. - Ci. Exatas Terra, Ci. Agr. Eng., 14, p.43 (2008).

7. Walter, M.; Silva, L. P. \& Emanuelli, T. - Cien. Rural, 35, p.974 (2005).

8. Raupp, D. S.; Moreira, S. S.; Banzatto, D. A. \& Sgarbieri, V. C. - Cien. Tecnol. Alim., 19 (1999).

9. Costa, P. M.; Tavares, M. I. B.; Bathista, A. L. S.; Silva, E. O. \& Nogueira, J. S. - J. Appl. Polym. Sci., 105, p.973 (2007).

10. Oliveira, A. M. G.; Diniz, M. S. \& Caldas, R. C. - "Comunicado Técnico 119 - Embrapa Mandioca e Fruticultura" (2006).

11. Macomber, R. S. - "A complete introduction to modern NMR spectroscopy", 2nd ed, John Wiley and Sons, New York (1998).

12. Breitmaier, E. \& Voelter, W. - "Carbon-13 NMR Spectroscopy", 3rd ed., VCh, Weinheim, Germany (1987). 
13. Costa, P. M. "Caracterização de amidos extraídos de sementes de frutas tropicais por espectroscopia de ressonância magnética nuclear", Dissertação de Mestrado, Instituto de macromoléculas, Universidade Federal do Rio de Janeiro, Brasil (2008).

14. Tavares, M. I. B.; Bathista, A. L. B. S.; Silva, E. O.; Costa, P. M.; Filho, N. P. \& Nogueira, J. S. - J. Appl. Polym. Sci., 92, p.2151 (2004).

15. Bello, M.; Ochoa, N.; Balsamo, V.; López-Carrasquero, F.; Coll, S.; Monsalve, A. \& González, G. - Carboh. Polym. (2010).

16. Corradini, E.; Lotti, C. \& Medeiros, E. S. Polímeros, 15, p.268 (2005).
17. Chi, H.; Xu, K.; Wu, X.; Chen, Q.; Xue, D.; Song, C.; Zhang, W. \& Wang, P. - Food Chem., 106, p.923 (2008).

18. Costa, P. M.; Tavares, M. I. B.; Silva, E. O.; Bathista, A. L. B. S.; Nogueira, J. S.; Ferreira, A. G.; Barison, A.; Daolio, C. \& Vizzoto, L. Inter. J. Polym. Mater., 56, p.1135 (2007).

Enviado: $11 / 03 / 10$

Reenviado: $13 / 09 / 10$

Aceito: $22 / 09 / 10$

DOI: $10.1590 / \mathrm{S} 0104-14282011005000032$ 